\begin{tabular}{|c|l|}
\hline Title & Nonuniform site charge distribution and fluctuations of charge order in the metallic state of a -(BEDT-TTF)213 \\
\hline Author(s) & $\begin{array}{l}\text { Yue, Yue; Y amamoto, Kaoru; Uruichi, Mikio; Nakano, Chikako; Y akushi, Kyuya; Y amada, Shigeaki; Hiejima, } \\
\text { Toshihiro; Kawamoto, A tsushi }\end{array}$ \\
\hline Citation & $\begin{array}{l}\text { Physical Review B, 82(7), 075134 } \\
\text { https://doi.org/10.1103/PhysRevB.82.075134 }\end{array}$ \\
\hline Issue Date & 2010-08_15 \\
\hline Doc URL & http://hdl.handle.net/2115/43848 \\
\hline Rights & @2010 The A merican Physical Society \\
\hline Type & article \\
\hline File Information & PRB82-7_075134.pdf \\
\hline
\end{tabular}

Instructions for use 


\title{
Nonuniform site-charge distribution and fluctuations of charge order in the metallic state of $\alpha$-(BEDT-TTF $)_{2} \mathbf{I}_{3}$
}

\author{
Yue Yue, Kaoru Yamamoto, Mikio Uruichi, Chikako Nakano, and Kyuya Yakushi \\ Institute for Molecular Science, Graduate University for Advanced Studies, 38 Nishigonaka, Myodaiji, Okazaki, Aichi 444-8585, Japan \\ Shigeaki Yamada and Toshihiro Hiejima \\ Department of Nanochemistry, Faculty of Engineering, Tokyo Polytechnic University, 1583 Iiyama, Atsugi 243-0297, Japan
}

\author{
Atsushi Kawamoto \\ Department of Physics, Hokkaido University, Kita-Ku, Sapporo 060-0810, Japan
}

(Received 2 March 2010; published 26 August 2010)

\begin{abstract}
We present the site-charge distribution in the charge-ordered and metallic states of $\alpha$-(BEDT-TTF ${ }_{2} \mathrm{I}_{3}$, based on the assignment of the $\mathrm{C}=\mathrm{C}$ stretching modes $\nu_{2}, \nu_{3}$, and $\nu_{27}$ of $\alpha$-(BEDT-TTF ${ }_{2} \mathrm{I}_{3}$ with the aid of ${ }^{13} \mathrm{C}$ - and deuterium-substituted compounds. The nonuniform site charges in both the metallic and insulating phases were consistent with those determined by a recent x-ray diffraction study. Comparing the line shapes of the chargesensitive vibrational modes of $\alpha$-(BEDT-TTF $)_{2} \mathrm{I}_{3}$ with those of isostructural $\alpha$-(BEDT-TTF $)_{2} \mathrm{NH}_{4} \mathrm{Hg}(\mathrm{SCN})_{4}$, we propose a thermally activated fluctuation of charge order in the metallic phase of $\alpha$-(BEDT-TTF $)_{2} \mathrm{I}_{3}$. This fluctuation was considerably suppressed above $0.65 \mathrm{GPa}$. The optical conductivity in the metallic phase shows no Drude response.
\end{abstract}

DOI: 10.1103/PhysRevB.82.075134

PACS number(s): 78.30.- $\mathrm{j}, 78.20 .-\mathrm{e}$

\section{INTRODUCTION}

Among the various known organic conductors, ${ }^{1,2}$ $\alpha-(\mathrm{BEDT}-\mathrm{TTF})_{2} \mathrm{I}_{3}[\mathrm{BEDT}-\mathrm{TTF}=$ bis(ethylenedithio)tetrathiafulvalene, hereafter BEDT-TTF is abbreviated as ET] shows rich solid-state properties such as charge ordering, ${ }^{3}$ superconductivity, ${ }^{4}$ a zero-gap state ${ }^{5}$ persistent photoconduction, ${ }^{6}$ a photoinduced phase transition, ${ }^{7}$ and a nonlinear optical response. ${ }^{8} \alpha$ - $(\mathrm{ET})_{2} \mathrm{I}_{3}$ was synthesized more than a quarter century ago. ${ }^{9}$ The structure of $\alpha$-(ET) ${ }_{2} \mathrm{I}_{3}$ consists of alternating anion and donor layers with a unit cell accommodating four ET molecules. The band calculation predicts a two-dimensional semimetal with electron and hole pockets. ${ }^{10}$ $\alpha$-(ET) $)_{2} \mathrm{I}_{3}$ exhibits a first-order metal-insulator (MI) phase transition at $T_{\mathrm{MI}}=135 \mathrm{~K} .{ }^{10}$ The $\mathrm{MI}$ transition is considered to be driven mainly by on-site and intersite Coulomb interaction. Based on theoretical, ${ }^{11-13}{ }^{13} \mathrm{C}-\mathrm{NMR},{ }^{3,14} \mathrm{Raman},{ }^{15}$ and $\mathrm{X}$-ray ${ }^{16}$ studies, the insulating phase is regarded as a chargeordered state involving a moderate structural change. The charge-ordered state can be characterized by its ordered pattern and amplitude. The long-range charge order is often accompanied by a change in symmetry. The breaking of symmetry was first suggested by an x-ray diffraction experiment. ${ }^{17}$ After the idea of charge order $(\mathrm{CO})$ in this $\mathrm{MI}$ transition was introduced, symmetry breaking in the insulating phase was confirmed by Raman spectroscopy. ${ }^{15}$ An x-ray diffraction experiment utilizing anomalous scattering effects supported the Raman results. ${ }^{16}$ Decisive evidence for the breaking of inversion symmetry was given by a second harmonic generation $(\mathrm{SHG})$ experiment. ${ }^{8}$

Regarding the amplitude (site-charge distribution) of the charge order, Heidmann et al. ${ }^{18}$ proposed a possible rearrangement of the site-charge distribution at temperatures below $T_{\mathrm{MI}}$ based on experimental investigations of the thermal expansion coefficient and ac calorimetry. ${ }^{19}$ More quantitative consideration of the site-charge distribution was conducted on the basis of the infrared spectrum of a powdered sample. ${ }^{20}$ However, the site-charge distribution in the insulating state $\left(T<T_{\mathrm{MI}}\right)$ was very different from the results of a recent $\mathrm{x}$-ray diffraction study, which estimated the site charges through the geometry of ET molecules. ${ }^{16}$ This discrepancy likely arose from ambiguity in the assignment of the infrared-active bands, which were screened by strong electronic absorption. The Raman experiment showed a drastic change in charge disproportionation amplitude. ${ }^{15}$ However, the estimation of the amplitude was still inaccurate, because the relationship between the site charge $\rho$ and the frequency $\nu(\rho)$ of $\mathrm{ET}^{\rho+}$ had not been well established. Later, more reliable relationships were presented for both the Raman-active and infrared-active modes. ${ }^{21}$ As unambiguous assignment is crucial in this kind of spectroscopic investigation, rational assignment was conducted with the aid of single crystals of ${ }^{13} \mathrm{C}$ - and deuterium-substituted ET salts. The first purpose of this study is to evaluate the $\mathrm{CO}$ amplitude in the insulating phase, and to examine the results of the previous infrared $^{18}$ and $\mathrm{x}$-ray diffraction ${ }^{16}$ studies.

Compared with the insulating phase, the metallic phase of $\alpha$-(ET) ${ }_{2} \mathrm{I}_{3}$ has not been thoroughly investigated, although the electronic structure of the metallic phase is related to the superconductivity under uniaxial strain, zero-gap state under hydrostatic pressure, and the excited state of the photoinduced metal-insulator transition. The metallic phase at ambient pressure has several peculiar properties. For example, the electrical resistivity is nearly temperature independent. ${ }^{22}$ The optical behavior in the far-infrared region is not metal-like; the dielectric constant is positive below $10 \mathrm{~cm}^{-1}$, and the optical conductivity has no Drude-type response. ${ }^{23,24} \mathrm{We}$ speculate that the fluctuation of $\mathrm{CO}$ is associated with these unusual metallic states. In contrast to $\theta-(\mathrm{ET})_{2} \mathrm{RbZn}(\mathrm{SCN})_{4},{ }^{25}$ it is difficult to detect fluctuations of $\mathrm{CO}$ by $\mathrm{x}$-ray diffraction 
because the unit cell of the $\mathrm{CO}$ phase is the same as that of the metallic phase except for the loss of inversion symmetry. The second purpose of this study is to investigate the fluctuations of $\mathrm{CO}$ in the metallic phase through analysis of the linewidths of the charge-sensitive modes. In this context, we also measured the temperature dependence of optical conductivity.

\section{EXPERIMENTAL TECHNIQUES}

Single crystals of $\alpha$-(ET) ${ }_{2} \mathrm{I}_{3}$ were grown by an electrochemical oxidation method in a tetrahydrofuran solution of ET $\left({ }^{13} \mathrm{C}\right.$-substituted ET, and deuterium-substituted ET) and tetrabuthylammonium triiodide $\left[(t-\mathrm{Bu})_{4} \mathrm{NI}_{3}\right]$. In ${ }^{13} \mathrm{C}$ substituted ET, the carbon atoms at the central $\mathrm{C}=\mathrm{C}$ bridge were substituted by ${ }^{13} \mathrm{C}$. In deuterium-substituted ET, all hydrogen atoms in ethylene end groups were substituted by deuterium. In the following discussion, the charge-transfer salt of ${ }^{13} \mathrm{C}$ (deuterium-) substituted ET is denoted as $\alpha-\left({ }^{13} \mathrm{C}-\mathrm{ET}\right)_{2} \mathrm{I}_{3}\left(\alpha-\left(d_{8}-\mathrm{ET}\right)_{2} \mathrm{I}_{3}\right)$. The thin platelike crystals had a well-developed (001) face, which was parallel to the conducting layer of the compound. The crystal face and axes were determined by $\mathrm{x}$-ray diffraction using a Rigaku mercury charge coupled device diffractometer.

A polarized reflection spectrum in the region of 600-25 $000 \mathrm{~cm}^{-1}$ was obtained using two spectrometers and a common microscope (Spectratech IR-Plan). A Nicolet Nexus 870 FT-IR spectrometer $\left(4 \mathrm{~cm}^{-1}\right.$ resolution) was used for the 600-11000 $\mathrm{cm}^{-1}$ region, and an Atago Macs320 multichannel detection system was used for the $11000-25000 \mathrm{~cm}^{-1}$ region. For the low-temperature experiment, a small goniometer head was attached to the cold head of the cryostat (Oxford CF1104s), which was fixed to an XYZ stage. The details of the experimental methods have been described previously. ${ }^{26}$ The reflection and transmission spectra in the far-infrared region $\left(30-650 \mathrm{~cm}^{-1}\right)$ were obtained using a Bruker IFS-66v spectrometer $\left(2 \mathrm{~cm}^{-1}\right.$ resolution) combined with a cryostat Oxford OptistatCF. The reflectivity data were collected against a gold mirror in the sample chamber. After the collection of all data, gold was evaporated on the sample crystal without removing the crystal from the sample holder. The reflectivity of the goldcoated sample crystal was measured against a gold mirror to calibrate for $100 \%$ reflectivity. The experimental details were previously described elsewhere. ${ }^{27}$ We connected the reflectivity curves between far-infrared and mid-infrared regions at $650 \mathrm{~cm}^{-1}$. There were gaps of $10 \%$ and $5 \%$ in the reflectivity of the $b$ - and $a$-polarized directions, respectively. We adjusted the mid-infrared reflectivity to the far-infrared reflectivity since the reflectivity in the far-infrared region was calibrated using the gold-coated sample crystal. The reflectivity below $50 \mathrm{~cm}^{-1}$ was extrapolated using the HagenRubens equation above $136 \mathrm{~K}$, and a constant value below $134 \mathrm{~K}$. The room-temperature reflectivity in the visible region was used as the extrapolation curve for the lowtemperature reflectivity curves. Optical conductivity spectra were calculated from the reflection spectra by performing a Kramers-Kronig analysis.

Raman spectra were obtained using a Renishaw Ramascope System-1000 in backscattering geometry. HeNe (632.8

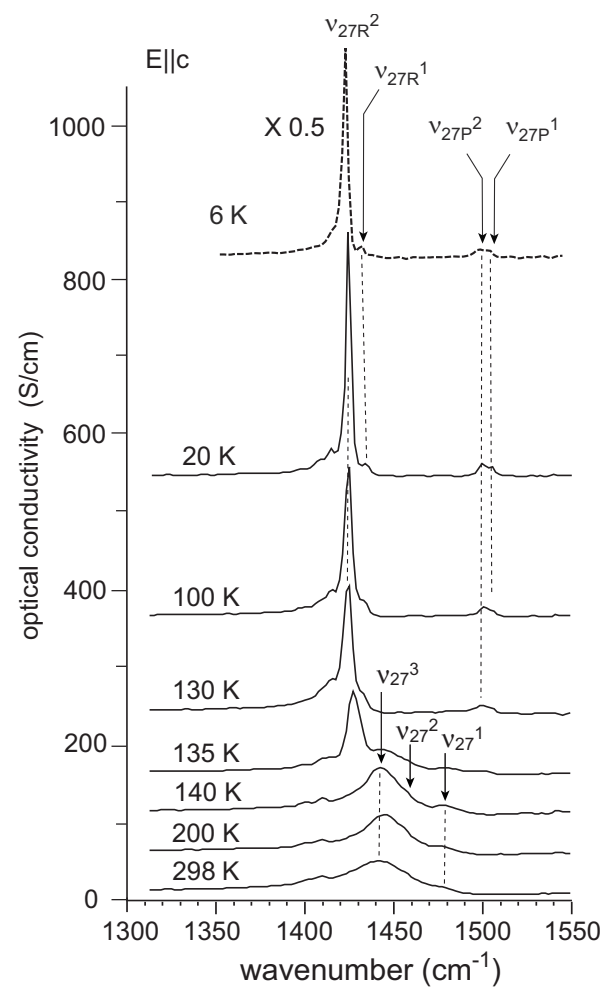

FIG. 1. Temperature dependence of the optical conductivity polarized along the $c$ direction, which is nearly perpendicular to the conducting layer. The broken line shows the $E \|_{c}$ spectrum of $\alpha-\left(d_{8}-\mathrm{ET}\right)_{2} \mathrm{I}_{3}$ measured at $6 \mathrm{~K}$. For $\alpha-(\mathrm{ET})_{2} \mathrm{I}_{3}$, the frequency of $\nu_{27 \mathrm{P}}^{1}, \nu_{27 \mathrm{P}}^{2}, \nu_{27 \mathrm{R}}^{1}$, and $\nu_{27 \mathrm{R}}^{2}$ are $1506 \mathrm{~cm}^{-1}, 1501 \mathrm{~cm}^{-1}, 1435 \mathrm{~cm}^{-1}$, and $1425 \mathrm{~cm}^{-1}$, respectively.

$\mathrm{nm})$ and $\operatorname{Ar}(514.5 \mathrm{~nm})$ lasers were used as the excitation light, and their beams were focused on an area $\sim 50 \mu \mathrm{m}$ in diameter at powers in the range 10-80 $\mu \mathrm{W}$. Since the crystal was triclinic, an analyzer was not used for the scattered light. In this paper, only the polarization of the excitation radiation was used to denote the polarization configuration of Raman spectra (e.g., $E \|_{c}$ ). Details of the low-temperature experiment including the high-pressure technique were described elsewhere. ${ }^{15,28}$

\section{RESULTS AND DISCUSSION}

\section{A. Amplitude of charge order in low-temperature insulating phase}

Figure 1 shows the temperature dependence of the $E \|_{c}$ optical conductivity. Since the $c$ axis is nearly perpendicular to the conducting plane, the vibrational modes can be observed free from the screening effect by low-frequency electronic excitation. The temperature dependence of the frequency and linewidth [full width at half maximum (FWHM)] are displayed in Fig. 2. As shown in Fig. 1, the spectrum shows a drastic change at the MI transition temperature $\left(T_{\mathrm{MI}}=135 \mathrm{~K}\right)$. The low-temperature phase is well established as the charge-ordered state, in which localized charges form a horizontal stripe breaking inversion symmetry. Below $T_{\mathrm{MI}}$, the broad band splits into two groups, and each group 


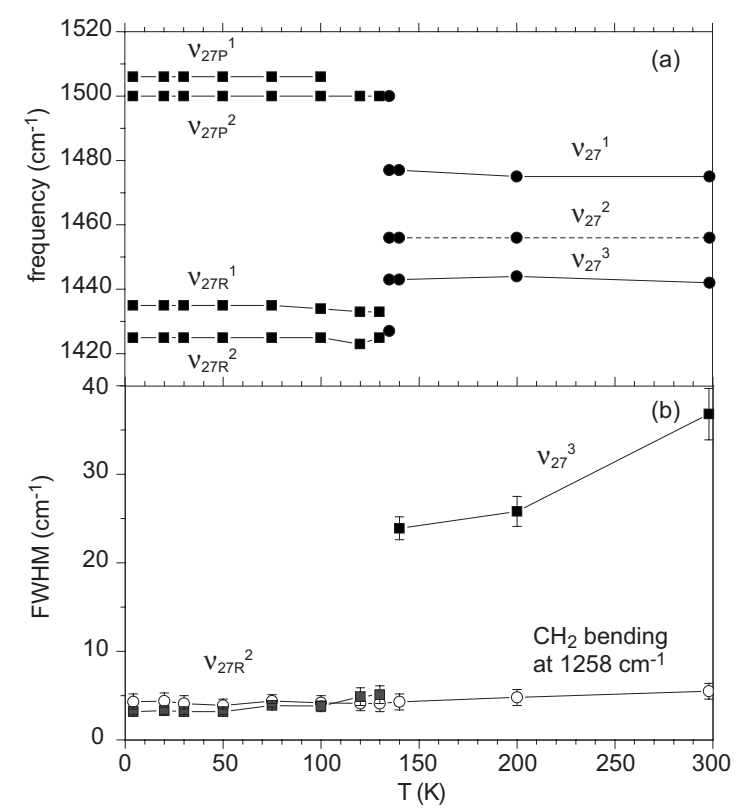

FIG. 2. (a) Frequencies of $\nu_{27}$ modes plotted against temperature. (b) Temperature dependence of the linewidth (FWHM) of the $\nu_{27}^{3}$ (black square) mode, which involves weak $\nu_{27}^{2}$ and $\nu_{27 \mathrm{R}}^{2}$ (gray square) modes. The temperature dependence of the $\mathrm{CH}_{2}$ bending mode (open circle) at $1258 \mathrm{~cm}^{-1}$, the frequency of which is insensitive to site charge, is shown for comparison.

consists of two vibrational bands. These four bands are unambiguously assigned to $\nu_{27 \mathrm{R}}^{\mathrm{j}}$ and $\nu_{27 \mathrm{P}}^{\mathrm{j}}(j=1,2)$ through the comparison with $\alpha-\left(d_{8}-\mathrm{ET}\right)_{2} \mathrm{I}_{3} .{ }^{29}$ The $\nu_{27 \mathrm{R}}^{\mathrm{j}}$ and $\nu_{27 \mathrm{P}}^{\mathrm{j}}$ modes are the out-of-phase ring $\mathrm{C}=\mathrm{C}$ stretching mode at chargerich and charge-poor sites, respectively. There is a big difference in the intensity of the $\nu_{27}$ mode at charge-rich sites. We interpreted that the strong $\nu_{27 \mathrm{R}}$ modes interacts with each other through dipole-dipole interactions. The nearly in-phase mode between the interacting $\nu_{27}$ has a strong intensity, because the vibrationally induced dipoles are parallel to each other, whereas the nearly out-of-phase mode had an extremely weak intensity, because the induced dipoles are antiparallel. Since the intensity of $\nu_{27 \mathrm{P}}$ is weak, the dipoledipole interaction is also weak, causing their intensities to be comparable.

The unit cell of $\alpha$-(ET) ${ }_{2} \mathrm{I}_{3}$ contains four ET molecules. If the space group of the unit cell is $P \overline{1}$, two molecules (B and C) are located at the center of symmetry, and the other two (A and $\mathrm{A}^{\prime}$ ) are connected by the center of symmetry (see Fig. 3 of Ref. 16 for the definition of A, A', B, and C). Therefore, three modes of $\nu_{27}$ are infrared active and one mode of $\nu_{27}$ is Raman active. As shown Fig. 1, four $\nu_{27}$ modes were observed in the optical conductivity. This observation provides clear evidence for the breaking of the center of symmetry. The symmetry breaking in the CO state is consistent with $\mathrm{x}$-ray diffraction ${ }^{16}$ and SHG (Ref. 8) studies. Since the $\nu_{27}$ mode does not couple with the electronic excitation, the frequency directly reflects the site charge. If we apply the linear relationship $\nu_{27}(\rho)=1538-140 \rho(0<\rho<1)$ (Ref. 21) to the frequencies of $\nu_{27}$ shown in the figure caption of Fig. 1, the site-charge $(\rho)$ distribution was estimated as $\left(0.8_{1}, 0.7_{4}, 0.2_{6}\right.$, and $\left.0.2_{3}\right) .^{30}$ This result was different from

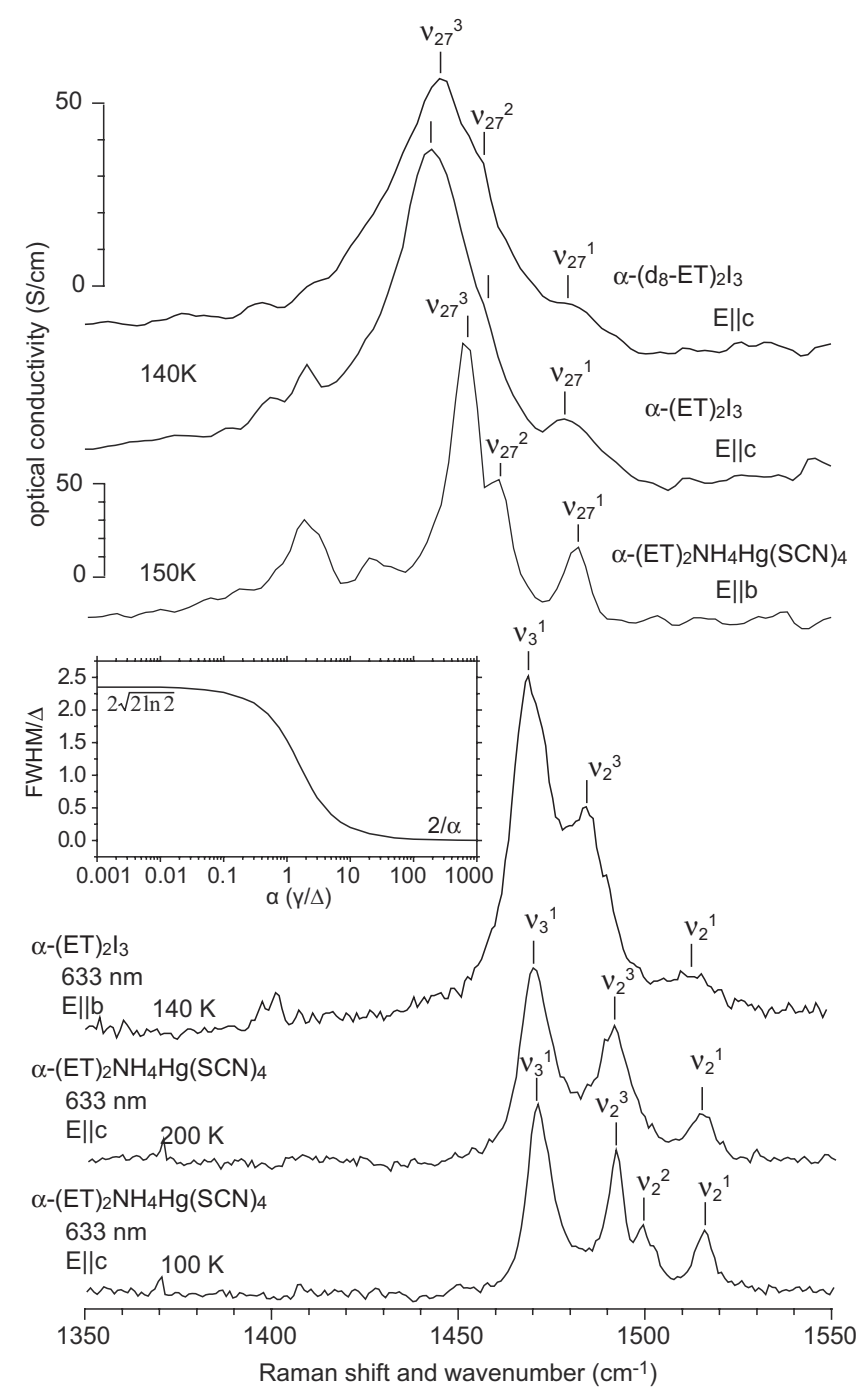

FIG. 3. Spectral shape of the $\nu_{27}, \nu_{3}$, and $\nu_{2}$ modes of $\alpha-(\mathrm{ET})_{2} \mathrm{I}_{3}$ and $\alpha-(\mathrm{ET})_{2} \mathrm{NH}_{4} \mathrm{Hg}(\mathrm{SCN})_{4}$. The assignment for $\nu_{3}$ and $\nu_{2}$ shown in this figure was supported by the isotope shift in the ${ }^{13} \mathrm{C}$-substituted compounds. The inset shows the numerical calculation of full width at half maximum divided by $\Delta(\mathrm{FWHM} / \Delta)$ plotted against $\alpha$ in the Gaussian modulation model. See text for the definition of $\Delta$ and $\alpha$.

the values $(0.9,0.52,0.52$, and 0.15$)$, estimated from the $\nu_{27}$ mode of the powdered sample ${ }^{20}$ but agrees well with the site-charge distribution $\left(\mathrm{A}=0.8_{2}, \mathrm{~B}=0.7_{3}, \mathrm{~A}^{\prime}=0.2_{9}\right.$, and $\mathrm{C}$ $=0.26$ ) estimated from the $\mathrm{X}$-ray diffraction experiment at 20 $\mathrm{K}$. As shown in Fig. 2(a), the site charge is temperature independent in contrast to the result of $\mathrm{x}$-ray diffraction experiment.

\section{B. Nonuniform site-charge distribution in the metallic phase}

Figure 3 shows the infrared- and Raman-active chargesensitive modes $\left(\nu_{27}\right.$ and $\left.\nu_{2}\right)$ in the metallic phases of $\alpha$-(ET) $)_{2} \mathrm{I}_{3}$ and $\alpha$ - $\left(d_{8}-\mathrm{ET}\right)_{2} \mathrm{I}_{3}$. For the purpose of comparison, Fig. 3 also shows the corresponding spectra of isostructural $\alpha$-(ET) ${ }_{2} \mathrm{NH}_{4} \mathrm{Hg}(\mathrm{SCN})_{4}$, which has a wider bandwidth. ${ }^{31}$ The frequencies of these modes are listed in Table I. The $\nu_{27}$ mode of $\alpha$-(ET) ${ }_{2} \mathrm{I}_{3}$ consists of three bands. The bands at 
TABLE I. Frequencies of charge-sensitive modes in the metallic phase. The numerical value in parenthesis represents the linewidth (FWHM).

\begin{tabular}{|c|c|c|c|}
\hline & \multirow{2}{*}{ 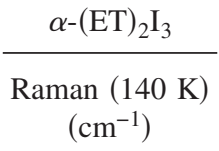 } & \multicolumn{2}{|c|}{$\alpha-(\mathrm{ET})_{2} \mathrm{NH}_{4} \mathrm{Hg}(\mathrm{SCN})_{4}$} \\
\hline & & $\begin{array}{c}\underset{\left(\mathrm{cm}^{-1}\right)}{\operatorname{Raman}(200 \mathrm{~K})} \\
\end{array}$ & $\begin{array}{c}\operatorname{Raman}(100 \mathrm{~K}) \\
\left(\mathrm{cm}^{-1}\right)\end{array}$ \\
\hline \multirow{5}{*}{$\begin{array}{l}\nu_{2}^{1} \\
\nu_{2}^{2} \\
\nu_{2}^{3}\end{array}$} & $1515(21)$ & $1515(8)$ & $1516(5)$ \\
\hline & & & $1500(5)$ \\
\hline & 1485 (14) & $1491(13)$ & $1492(6)$ \\
\hline & $\alpha-(\mathrm{ET})_{2} \mathrm{I}_{3}$ & $\alpha-\left(d_{8}-\mathrm{ET}\right)_{2} \mathrm{I}_{3}$ & $\alpha-(\mathrm{ET})_{2} \mathrm{NH}_{4} \mathrm{Hg}(\mathrm{SCN})_{4}$ \\
\hline & $\begin{array}{l}\text { IR }(140 \mathrm{~K}) \\
\quad\left(\mathrm{cm}^{-1}\right)\end{array}$ & $\begin{array}{l}\text { IR }(140 \mathrm{~K}) \\
\left(\mathrm{cm}^{-1}\right)\end{array}$ & $\begin{array}{l}\text { IR }(150 \mathrm{~K}) \\
\quad\left(\mathrm{cm}^{-1}\right)\end{array}$ \\
\hline$\nu_{27}^{1}$ & 1477 (18) & 1477 & $1482(5)$ \\
\hline$\nu_{27}^{2}$ & & $\sim 1456[\mathrm{sh}]$ & $1462(4)$ \\
\hline$\nu_{27}^{3}$ & $1443(25)$ & 1444 & $1453(10)$ \\
\hline
\end{tabular}

$1477 \mathrm{~cm}^{-1}$ and $1443 \mathrm{~cm}^{-1}$ in the $140 \mathrm{~K}$ spectrum were assigned to $v_{27}^{1}$ and $\nu_{27}^{3}$, respectively, and $\nu_{27}^{2}$ appeared to be an inflection point between $v_{27}^{1}$ and $\nu_{27}^{3}$. Indeed, $\nu_{27}^{2}$ appeared more clearly as a shoulder at $\sim 1456 \mathrm{~cm}^{-1}$ in $\alpha-\left(d_{8}-\mathrm{ET}\right)_{2} \mathrm{I}_{3}$. In the case of $\alpha$ - $(\mathrm{ET})_{2} \mathrm{NH}_{4} \mathrm{Hg}(\mathrm{SCN})_{4}$, the $\nu_{27}^{2}$ mode was observed most clearly at $1462 \mathrm{~cm}^{-1}$. In both compounds, therefore, the $\nu_{27}$ mode was split into three, which means that the three nonequivalent sites in the unit cell had nonuniform site charges. Using the spectra measured at 140 and $150 \mathrm{~K}$, the site-charge distributions were roughly estimated to be $\left(0.6_{8}\right.$, $\sim 0.6$, and $\left.0.4_{4}\right)$ for $\alpha$ - $(\mathrm{ET})_{2} \mathrm{I}_{3}$ and $\left(0.6_{2}, 0.5_{6}\right.$, and $\left.0.4_{0}\right)$ for $\alpha$-(ET) ${ }_{2} \mathrm{NH}_{4} \mathrm{Hg}(\mathrm{SCN})_{4}$. In the Raman spectrum, the $\nu_{2}^{2}$ mode was buried by the $\nu_{2}^{3}$ mode at $140 \mathrm{~K}$ in $\alpha-(\mathrm{ET})_{2} \mathrm{I}_{3}$, as well as in the $200 \mathrm{~K}$ spectrum of $\alpha-(\mathrm{ET})_{2} \mathrm{NH}_{4} \mathrm{Hg}(\mathrm{SCN})_{4}$. The site charge distribution estimated from the $\nu_{2}$ mode of $\alpha$-(ET) ${ }_{2} \mathrm{I}_{3}$ was $\left(0.6_{8},-, 0.4_{3}\right),{ }^{21}$ which was consistent with the result estimated from $\nu_{27}$. The site-charge distribution in the metallic phase of $\alpha-(\mathrm{ET})_{2} \mathrm{I}_{3}$ also agreed with the estimation based on the x-ray diffraction study at $150 \mathrm{~K}$ (see Fig. 3 of Ref. 16).

The amplitude of the nonuniform site charge distribution in the metallic phase is much smaller than that in the $\mathrm{CO}$ phase. The reason for this nonuniform site-charge distribution has not been completely clarified but it has been reported to be a precursor to charge ordering in the lowtemperature phase. ${ }^{14}$ The site-charge distribution of $\alpha$ - $(\mathrm{ET})_{2} \mathrm{I}_{3}$ was estimated to be $\mathrm{B} \approx \mathrm{A}=\mathrm{A}^{\prime}>\mathrm{C}$ on the basis of the $\mathrm{X}$-ray diffraction study, ${ }^{16}$ whereas that of $\alpha-(\mathrm{ET})_{2} \mathrm{NH}_{4} \mathrm{Hg}(\mathrm{SCN})_{4}$ was regarded as $\mathrm{B} \approx \mathrm{C}>\mathrm{A}=\mathrm{A}^{\prime}$ on the basis of the NMR study. ${ }^{32}$ Both of these are different from the horizontal site-charge distribution, $\mathrm{A} \approx \mathrm{B}>\mathrm{A}^{\prime} \approx \mathrm{C}$, in the $\mathrm{CO}$ state of $\alpha-(\mathrm{ET})_{2} \mathrm{I}_{3}$. Meanwhile, a tight-binding calculation under the mean-field approximation predicts the site-charge distribution $\left(\mathrm{B}=0.6_{1}, \mathrm{~A}=\mathrm{A}^{\prime}=0.5_{1}\right.$, and $\left.\mathrm{C}=0.3_{8}\right)$ which agrees qualitatively with the observed one. ${ }^{33}$ Therefore, we believe that the nonuniform site-charge distribution in the metallic state is due to nonequivalent transfer integrals around the three nonequivalent sites in the unit cell. ${ }^{15}$ This interpretation implies that the electronic state of $\alpha-(\mathrm{ET})_{2} \mathrm{I}_{3}$ can be described by band picture, in other words, the electron wave function is effectively coherent. Therefore, the site charge and its fluctuation are derived from the character of the wave function of the valence electron of ET

Although $\quad \nu_{27}$ and $\nu_{2}$ of $\alpha$ - $(\mathrm{ET})_{2} \mathrm{I}_{3} \quad$ and $\alpha$ - $(\mathrm{ET})_{2} \mathrm{NH}_{4} \mathrm{Hg}(\mathrm{SCN})_{4}$ are split into three with similar split widths, the linewidth of each mode of $\alpha$-(ET) ${ }_{2} \mathrm{I}_{3}$ is very broad compared to those of $\alpha-(\mathrm{ET})_{2} \mathrm{NH}_{4} \mathrm{Hg}(\mathrm{SCN})_{4}$. As shown in Table I, the former's linewidths (FWHM) of $\nu_{27}^{1}$ and $\nu_{27}^{3}$ (including $\nu_{27}^{2}$ ) was $\sim 18 \mathrm{~cm}^{-1}$ and $\sim 25 \mathrm{~cm}^{-1}$, respectively, whereas the latter's were $\Delta \nu_{27}^{1}=5 \mathrm{~cm}^{-1}$ and $\Delta \nu_{27}^{3}$ (including $\nu_{27}^{2}$ ) $=10 \mathrm{~cm}^{-1}$. The linewidths of the Raman spectrum were estimated to be $\Delta \nu_{2}^{1}=21 \mathrm{~cm}^{-1}$ and $\Delta \nu_{2}^{3}$ $=14 \mathrm{~cm}^{-1}$ for $\alpha-(\mathrm{ET})_{2} \mathrm{I}_{3}$, and $\Delta \nu_{2}^{1}=8 \mathrm{~cm}^{-1}$ and $\Delta \nu_{2}^{3}$ $=13 \mathrm{~cm}^{-1}$ for $\alpha-(\mathrm{ET})_{2} \mathrm{NH}_{4} \mathrm{Hg}(\mathrm{SCN})_{4}(200 \mathrm{~K}){ }^{34}$ If we compare the linewidths of the rather isolated $\nu_{27}^{1}$ and $\nu_{2}^{1}$ modes, the linewidths of $\alpha$-(ET) ${ }_{2} \mathrm{I}_{3}$ are more than double those of $\alpha$-(ET) ${ }_{2} \mathrm{NH}_{4} \mathrm{Hg}(\mathrm{SCN})_{4}$, despite the lower temperature of the former spectrum than the latter. Since the linewidth in $\alpha$-(ET) $)_{2} \mathrm{I}_{3}$ is very broad compared to a charge-insensitive vibrational mode such as the $\mathrm{CH}_{2}$ bending mode [see Fig. 2(b)], we consider that the broad linewidth is associated with an inhomogeneous charge distribution at each site because the frequencies of $\nu_{27}$ and $\nu_{2}$ strongly depend upon the site charge $\left(140 \mathrm{~cm}^{-1} / e\right.$ for $\nu_{27}$ and $120 \mathrm{~cm}^{-1} / e$ for $\left.\nu_{2}\right){ }^{21}$ If we assume that the inhomogeneous charge distribution fluctuates dynamically, the fluctuation of site charge can be characterized by its amplitude or variance $\Delta_{c}=\left\langle\Delta \rho^{2}\right\rangle^{1 / 2}$ and correlation time $\tau=\int_{0}^{\infty}\left(\langle\Delta \rho(0) \Delta \rho(t)\rangle / \Delta_{c}^{2} d_{i}\right.$, where $\Delta \rho=\rho(\mathrm{t})-\rho_{0}$ is the time-dependent site-charge variation from the average site charge $\rho_{0}$. This charge fluctuation modulates the frequencies of $\nu_{27}$ and $\nu_{2}$ with an amplitude $\Delta_{\mathrm{f}}$ and correlation time $\tau$. If we assume that the site charge fluctuates stochastically as a Gaussian process, the line shape of the charge-sensitive mode is characterized by the parameter $\alpha=\left(\Delta_{\mathrm{f}} \tau\right)^{-1}=\gamma / \Delta_{\mathrm{f}},{ }^{35}$ where $\gamma=1 / \tau$ is the fluctuation rate. If we follow conventional line-shape analysis, $\Delta_{\mathrm{f}}$ can be estimated by the square root of the second moment of the vibrational spectrum. ${ }^{36} \mathrm{We}$ analyzed the line shape of the rather isolated $\nu_{2}^{1}$ mode. ${ }^{37}$ To obtain the second moment of $\nu_{2}^{1}$ for $\alpha$ - $(\mathrm{ET})_{2} \mathrm{I}_{3}$, we fitted the $140 \mathrm{~K}$ spectrum using three Lorentz functions, and subtracted the Lorentz functions with peaks at $1470\left(\nu_{3}^{1}\right)$ and $1486 \mathrm{~cm}^{-1}\left(\nu_{2}^{3}\right)$. The second moment of the $\nu_{2}^{1}$ band was obtained by numerically integrating from 1491 to $1520 \mathrm{~cm}^{-1} .{ }^{38}$ The square root of the second moment was estimated to be $\Delta_{\mathrm{f}}>\sim 9.1 \mathrm{~cm}^{-1}$. Therefore, the amplitude of the site-charge fluctuation was estimated to be $\Delta_{\mathrm{c}}>\sim 0.08$. Since the site charge should have been between 0 and 1 , $\Delta_{\mathrm{c}}<\sim 0.14,{ }^{39}$ and thus $\Delta_{\mathrm{f}}<\sim 17 \mathrm{~cm}^{-1}$. Therefore, $\Delta_{\mathrm{f}}$ was likely to have been in the range of $9-17 \mathrm{~cm}^{-1}$. The ratio between the linewidth (FWHM) and $\Delta_{\mathrm{f}}$ was estimated to be $\sim 2.3-1.2$. The ratio FWHM/ $\Delta_{\mathrm{f}}$ of the Gaussian modulation model was numerically calculated and is plotted against $\alpha$ in the inset of Fig. $3 .{ }^{35}$ In this figure, the line shape approaches a Gaussian curve when $\alpha \rightarrow 0$ and a Lorentzian curve when $\alpha \rightarrow \infty$. Using this figure, the fluctuation rate $(\gamma)$ was roughly estimated to be $\gamma \sim(0.1-1.5) \Delta_{\mathrm{f}} \sim 1-25 \mathrm{~cm}^{-1}$. This slow fluctuation rate suggests collective motion of site charge.

A similar broad linewidth in a conductive phase was reported for $\theta$-(ET) ${ }_{2} \mathrm{RbZn}(\mathrm{SCN})_{4}$, in which the FWHM of the 


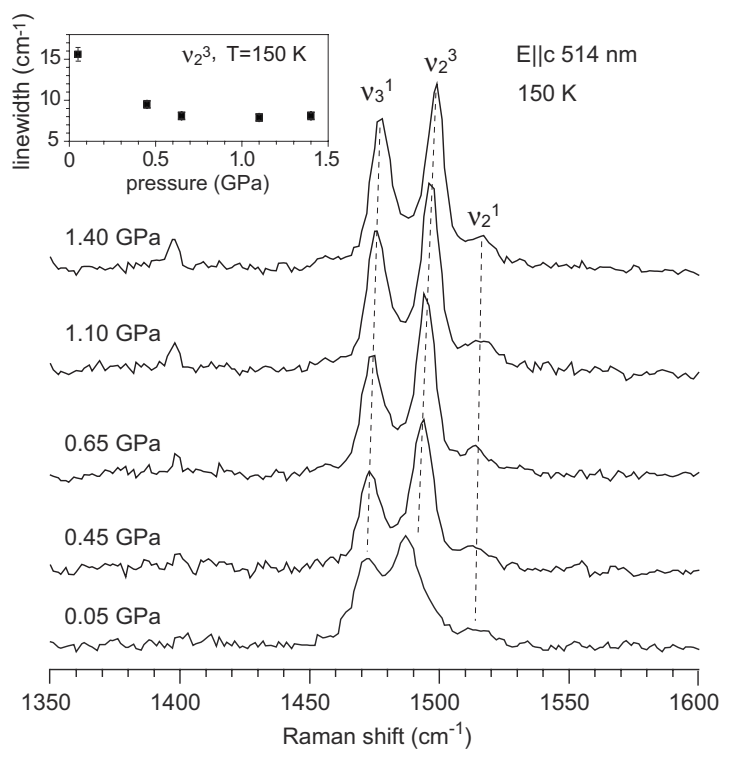

FIG. 4. Pressure dependence of the Raman spectrum of $\alpha$-(ET) ${ }_{2} \mathrm{I}_{3}$ measured at $150 \mathrm{~K}$. The $\nu_{3}$ and $\nu_{2}$ modes were significantly sharpened under hydrostatic pressure.

$\nu_{2}$ mode is $\sim 26 \mathrm{~cm}^{-1}$ at $200 \mathrm{~K}^{40}$ The electronic state of the conductive phase of this compound is regarded as a frustrated state, in which two-fold and three-fold short-range $\mathrm{CO}$ compete. ${ }^{41}$ This theoretical model is based on the observation of diffuse x-ray scattering by twofold and threefold superlattices. ${ }^{26}$ In the case of $\alpha$-(ET) ${ }_{2} \mathrm{I}_{3}$, however, $\mathrm{x}$-ray scattering by the superlattice has not been reported. Therefore, the only possible modes of short-range $\mathrm{CO}$ are horizontal and/or vertical stripes, if short-range CO exists. Using realistic parameters and taking electron-lattice coupling into account, Tanaka and Yonemitsu calculated the finitetemperature free energy for horizontal, vertical, and diagonal stripes and three fold $\mathrm{CO}$ of $\alpha-(\mathrm{ET})_{2} \mathrm{I}_{3}$ within the framework of the mean-field approximation. ${ }^{33}$ At low temperatures, these $\mathrm{CO}$ states and the paramagnetic metallic state are distributed within a narrow energy range $(\sim 10 \mathrm{meV}){ }^{42}$ Therefore, the free energy difference at finite temperatures in the metallic phase above $T_{\mathrm{MI}}$ is considerably reduced. ${ }^{33} \mathrm{We}$ therefore speculate that the metallic state of $\alpha-(\mathrm{ET})_{2} \mathrm{I}_{3}$ above $T_{\mathrm{MI}}$ involves short-range correlation of four horizontal and two vertical stripes (CO fluctuation). It should be noted that this $\mathrm{CO}$ fluctuation is enhanced as the temperature is increased. The slow fluctuation of site charge could be attributed to the dynamical fluctuation of inhomogeneously distributed short-range CO stripes. Such CO fluctuation would modulate the charge density at each site and broaden the linewidth of the charge-sensitive mode, if the fluctuation rate were sufficiently slow. Since the metallic state of $\alpha$-(ET) ${ }_{2} \mathrm{NH}_{4} \mathrm{Hg}(\mathrm{SCN})_{4}$ is more stable than $\alpha$ - $(\mathrm{ET})_{2} \mathrm{I}_{3}$ due to wider bandwidth, the short-range $\mathrm{CO}$ correlation at finite temperatures in the former compound seems to be more suppressed than in the latter. Even if short-range $\mathrm{CO}$ arises, the correlation length and amplitude may be much smaller, leading to a much higher fluctuation rate. This fast modulation narrows the linewidth of the charge-sensitive mode.

Although large hysteresis has not been observed, the MI transition of $\alpha$-(ET) ${ }_{2} \mathrm{I}_{3}$ is regarded as a first-order phase tran- sition, and thus the entropy change drives this MI transition. ${ }^{19}$ The thermally activated short-range $\mathrm{CO}$ correlation probably contributes to the enhancement of entropy in the metallic phase. As the temperature is lowered from room temperature to $T_{\mathrm{MI}}$, the linewidth of the $\nu_{27}$ mode narrows [see Fig. 2(b)]. It is reasonable that CO fluctuation is suppressed at lower temperatures, in contrast to a second-order phase transition, because the short-range $\mathrm{CO}$ correlation with high energy cannot be activated at low temperature. This is most likely not only due to the deactivation of short-range $\mathrm{CO}$ correlation, but also to lattice contraction, which increases transfer integrals more efficiently than intersite Coulomb energy. As it will be shown in Fig. 5, the optical conductivity increases with decreasing temperature, not only in the far-infrared region but also in the mid-infrared region, which implies an increase in the kinetic energy (transfer integrals). It is known that the hydrostatic pressure widens the bandwidth and suppresses the $\mathrm{CO}$ transition. ${ }^{22}$ To examine the influence of bandwidth (kinetic energy) on linewidth, a hydrostatic pressure experiment was conducted. Figure 4 shows the pressure dependence of the Raman spectrum of $\alpha$-(ET) ${ }_{2} \mathrm{I}_{3}$ at $150 \mathrm{~K}$. As shown in this figure, the linewidth of $\nu_{2}^{3}$, obtained by fitting a Lorentz function, decreased by almost half at $0.65 \mathrm{GPa}$, and was comparable to the linewidth of $\nu_{2}^{3}$ of $\alpha$ - $(\mathrm{ET})_{2} \mathrm{NH}_{4} \mathrm{Hg}(\mathrm{SCN})_{4}$ at $100 \mathrm{~K}$. The CO fluctuation should be greatly reduced at this pressure. We speculate that the $\mathrm{CO}$ fluctuation is even more suppressed in the zero-gap state at $1.8 \mathrm{GPa}^{5}$

\section{Optical conductivity}

Figure 5 shows the optical conductivity of $\alpha-(\mathrm{ET})_{2} \mathrm{I}_{3}$ polarized along the $b$ and $a$ axes in the conducting plane. The low-frequency region showed drastic changes in both polarizations at $T_{\mathrm{MI}}$. As shown in Fig. 5, a large optical gap $(\sim 0.1 \mathrm{eV})$ opens in the charge-ordered state. Meanwhile, a broad dip emerges at $\sim 2688 \mathrm{~cm}^{-1}$ in the $E \| b$ spectrum, which is assigned to the overtone mode of the strong vibronic mode of $\nu_{3}$ observed at $1349 \mathrm{~cm}^{-1}$. ${ }^{43}$ Figure 6 shows the $50 \mathrm{~K}$ optical conductivity spectrum below $600 \mathrm{~cm}^{-1}$. As we described in the Sec. III A, ET molecules are separated into two charge-rich and two charge-poor species in the charge-ordered state. Therefore, each intramolecular mode will be more or less split. As the $\nu_{9}\left(a_{\mathrm{g}}\right)$ and $\nu_{10}\left(a_{\mathrm{g}}\right)$ modes have relatively large electron-molecular-vibration coupling constants, ${ }^{44}$ the bands at 483,471 , and $469 \mathrm{~cm}^{-1}$ were assigned to the vibronic mode of $\nu_{9}$, and the bands at 456,451 , and $444 \mathrm{~cm}^{-1}$ were assigned to the vibronic $\nu_{10}$ modes. The broad bands at 409,404 , and $395 \mathrm{~cm}^{-1}$ were assigned to $\nu_{35}\left(b_{1 \mathrm{u}}\right){ }^{45}$ The interpretation of the low-frequency phonon bands is more difficult, as the unit cell contains four independent ET molecules and two independent $\mathrm{I}_{3}^{-}$ions, implying 33 optical phonons. ${ }^{46}$ In addition, the lattice modes are mixed with the low-frequency internal modes of ET. ${ }^{47}$ Here, we assigned the strong band at $123 \mathrm{~cm}^{-1}$, which was mainly polarized along the $a$ axis, to the antisymmetric stretching mode of $\mathrm{I}_{3}^{-}$. This assignment is supported by the polarization direction, and was confirmed by comparison with the spectrum of $\alpha^{\prime}$-(ET) ${ }_{2} \mathrm{IBr}_{2}$ (not shown). This is probably the same 

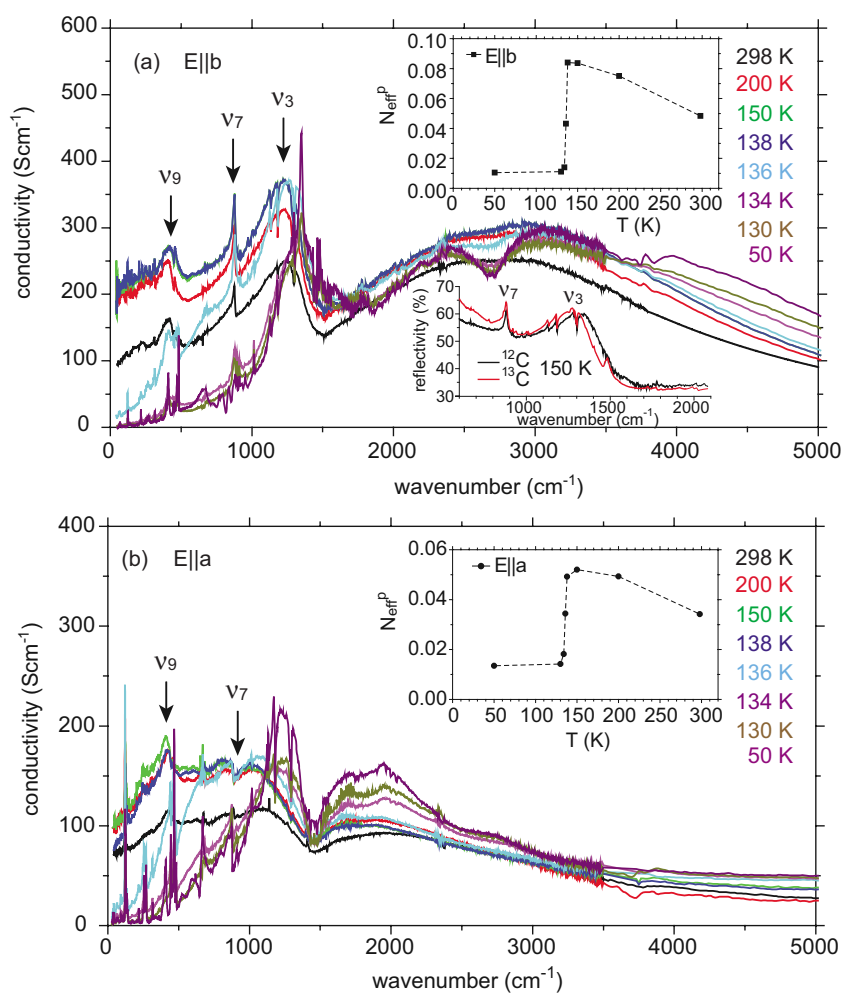

FIG. 5. (Color online) Temperature dependence of the optical conductivity of $\alpha$-(ET) ${ }_{2} \mathrm{I}_{3}$, measured on the (001) crystal face with the polarizations of $E \| b$ and $E \| a$. The inset shows the partial effective number of electrons with a cut-off frequency of $1000 \mathrm{~cm}^{-1}$. The inset in (a) shows the reflectivity curves of $\alpha-(\mathrm{ET})_{2} \mathrm{I}_{3}$ and $\alpha-\left({ }^{13} \mathrm{C}-\mathrm{ET}\right)_{2} \mathrm{I}_{3}$ in the spectral region of the $\nu_{3}$ and $\nu_{7}$ vibronic bands. Note that the broad band shows an isotope shift, whereas the vibrational bands show no isotope shift.

mode observed at 123 or $121 \mathrm{~cm}^{-1}$ in the Raman spectrum of $\alpha$-(ET) ${ }_{2} \mathrm{I}_{3} \cdot{ }^{48,49}$ This result may be associated with the broken symmetry and mixing with lattice modes. The lowfrequency region is shown in the inset with an expanded scale. The absorption spectra obtained from the transmission experiment agreed well with the optical conductivity spectra above $50 \mathrm{~cm}^{-1}$. Among several peaks observed below $50 \mathrm{~cm}^{-1}$, the peak at $31 \mathrm{~cm}^{-1}(E \| b)$ corresponds to a $31.6 \mathrm{~cm}^{-1}(E \| b)$ peak in the submillimeter conductivity reported by Zelezny et al. ${ }^{23}$ The peaks at $38 \mathrm{~cm}^{-1}(E \| b)$ and $65 \mathrm{~cm}^{-1}(E \| b)$ obtained by terahertz time-domain spectrometer ${ }^{50}$ may correspond to 40 and $68 \mathrm{~cm}^{-1}$. Dressel et $a l .{ }^{24}$ reported a strong band at $35 \mathrm{~cm}^{-1}(E\|a, E\| b)$ in the reflection spectrum at $60 \mathrm{~K}$. However, the corresponding peak was not observed in the transmission spectrum.

In the metallic phase, the gap region is filled by electronic excitation. The spectral weight in this region, represented by a partial effective number of electrons $\left(N_{e f f}^{p}\right)$ per formula unit, is plotted in the inset of Fig. 5. The partial effective number of electrons is defined as

$$
N_{e f f}^{p}=\frac{2 m}{\pi e^{2} N} \int_{0}^{\omega_{c}} \sigma(\omega) d \omega .
$$

Here, $N$ denotes the number of formula units $\left[\alpha-(\mathrm{ET})_{2} \mathrm{I}_{3}\right]$ per unit volume and the cut-off frequency was taken as $\omega_{\mathrm{c}}$

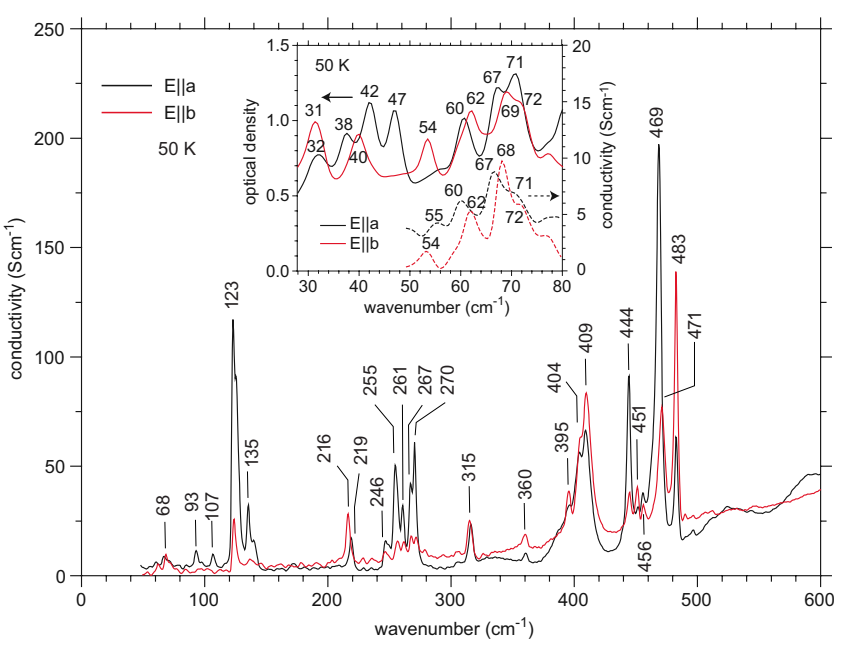

FIG. 6. (Color online) Optical conductivity in the far-infrared region measured on the $(001)$ crystal face at $50 \mathrm{~K}$ with the polarizations of $E \| b$ and $E \| a$. The inset shows the low-frequency region along with the absorption spectra obtained by transmission method using a single crystal with $\sim 35 \mu \mathrm{m}$ thickness.

$=1000 \mathrm{~cm}^{-1}$. The optical conductivity and spectral weight in this region continuously increased down to $T_{\mathrm{MI}}$, as shown in the insets of Fig. 5. The $138 \mathrm{~K}$ optical conductivity in the far-infrared region increased by about two times the roomtemperature value. This increase is consistent with the temperature dependence of the optical conductivity of Ref. 23 . In the same way as Ref. 23, the optical conductivity of the metallic phase is not Drude type, and the real part of the dielectric constant is positive down to $50 \mathrm{~cm}^{-1}$. The $\mathrm{CO}$ fluctuation described in the Sec. III B might be associated with this overdamped behavior of the Drude response. Meanwhile, $\alpha$-(ET) ${ }_{2} \mathrm{I}_{3}$ is a narrow-band semimetal with small Fermi surface pockets, in which the Fermi temperature measured from the top and bottom of the band is low compared with $T_{\mathrm{MI}}$. Therefore, decisive discussion is difficult. Prominent vibronic modes were also observed in the metallic phase: $\sim 1220 \mathrm{~cm}^{-1}(E \| b)$ assigned to $\nu_{3}, \sim 890 \mathrm{~cm}^{-1}$ (dip for $E \| a)$ and $876 \mathrm{~cm}^{-1}(E \| b)$ assigned to $\nu_{7}$, and $\sim 410 \mathrm{~cm}^{-1}(E \| a)$, and $\sim 415 \mathrm{~cm}^{-1}(E \| b)$ assigned to $\nu_{9} .{ }^{44}$ The very broad band at $\sim 1220 \mathrm{~cm}^{-1}(E \| b)$ appears to be an electronic transition. As shown in the inset of Fig. 5(a), however, the corresponding band of the ${ }^{13} \mathrm{C}$-substituted compound showed a clear redshift. This isotope shift strongly supports the assignment of the broad band as the $\nu_{3}$ vibronic mode. The results and assignment are consistent with the study of room-temperature optical conductivity reported by Meneghetti et al. $^{51}$

Finally, we will discuss the optical conductivity at $136 \mathrm{~K}$ which shows a spectral shape intermediate between metallic and insulating phases. As shown in Fig. 1, the $E \|_{\mathrm{c}}$ spectrum measured at $135 \mathrm{~K}$ consists of a sharp band characteristic of a $\mathrm{CO}$ phase, and a broad band characteristic of a metallic phase. ${ }^{52}$ These results suggest the coexistence of $\mathrm{CO}$ and metal domains at this temperature in the area of focused light. The Raman spectrum of $\alpha-\left({ }^{13} \mathrm{C}-\mathrm{ET}\right)_{2} \mathrm{I}_{3}$ was examined at around the transition temperature using $2 \mu \mathrm{m}$ spot of $\mathrm{Ar}^{+}$ laser. $^{53}$ The coexistent spectra were observed at 135 and 
135.5 $\mathrm{K}$ in both the cooling and heating processes. According to the preliminary mapping experiment, the spectrum corresponding to $\mathrm{CO}$, metal, and coexistent state was observed depending upon the measurement position. We therefore speculate that the spectra measured at $136 \mathrm{~K}$ (Fig. 5) and $135 \mathrm{~K}$ (Fig. 1) correspond to the coexistent state at the transition temperature of the first-order phase transition.

\section{CONCLUSION}

Combining the results of infrared-active charge-sensitive band with $\mathrm{x}$-ray diffraction and ${ }^{13} \mathrm{C} \mathrm{NMR}$ results, the sitecharge distribution of $\alpha$ - $(\mathrm{ET})_{2} \mathrm{I}_{3}$ was estimated to be (B $=0.6_{8}, \mathrm{~A}=\mathrm{A}^{\prime} \sim 0.6$, and $\left.\mathrm{C}=0.4_{4}\right)$ in metallic state and (A $\left.=0.8_{1}, \mathrm{~B}=0.7_{4}, \mathrm{~A}^{\prime}=0.2_{6}, \mathrm{C}=0.2_{3}\right)$ in $\mathrm{CO}$ state. The broad linewidths of the $\nu_{27}$ and $\nu_{2}$ modes of $\alpha$-(ET) $)_{2} \mathrm{I}_{3}$ in the metallic phase originated from slow $\left(1-25 \mathrm{~cm}^{-1}\right)$ fluctuations of the charge distribution at each site, which was associated with the thermal activation of short-range correlation of $\mathrm{CO}$ stripes. This CO fluctuation is suppressed by hydrostatic pressure. In the temperature dependence of the $\nu_{27}$ modes, we found a coexistence state of metal and $\mathrm{CO}$ domains, which shows that the MI transition at $135 \mathrm{~K}$ is the first-order phase transition.

\section{ACKNOWLEDGMENTS}

We thank S. Iwai of Tohoku University and Y. Tanaka of Institute for Molecular Science for helpful discussion. This work was partly supported by a Grant-in-Aid for Scientific Research (Grant No. 19350074) from MEXT, Japan.
${ }^{1}$ P. Batail, Chem. Rev. 104, 4887 (2004).

${ }^{2}$ J. Wosnitza, Fermi Surfaces of Low-Dimensional Organic Metals and Superconductors (Springer, New York, 1996).

${ }^{3}$ Y. Takano, K. Hiraki, H. M. Yamamoto, T. Nakamura, and T. Takahashi, Synth. Met. 120, 1081 (2001).

${ }^{4}$ N. Tajima, A. Ebina-Tajima, M. Tamura, Y. Nishio, and K. Kajita, J. Phys. Soc. Jpn. 71, 1832 (2002).

${ }^{5}$ N. Tajima, S. Sugawara, M. Tamura, R. Kato, Y. Nishio, and K. Kajita, EPL 80, 47002 (2007).

${ }^{6}$ N. Tajima, J. Fujisawa, N. Naka, T. Ishihara, R. Kato, Y. Nishio, and K. Kajita, J. Phys. Soc. Jpn. 74, 511 (2005).

${ }^{7}$ S. Iwai, K. Yamamoto, F. Hiramatsu, H. Nakaya, Y. Kawakami, and K. Yakushi, Phys. Rev. B 77, 125131 (2008).

${ }^{8}$ K. Yamamoto, S. Iwai, S. Boyko, A. Kashiwazaki, F. Hiramatsu, C. Okabe, N. Nishi, and K. Yakushi, J. Phys. Soc. Jpn. 77, 074709 (2008).

${ }^{9}$ K. Bender, I. Henning, D. Schweitzer, K. Dietz, H. Endres, and H. J. Keller, Mol. Cryst. Liq. Cryst. 108, 359 (1984).

${ }^{10}$ T. Mori, A. Kobayashi, Y. Sasaki, H. Kobayashi, G. Saito, and H. Ionokuchi, Chem. Lett. 13, 957 (1984).

${ }^{11}$ H. Kino and H. Fukuyama, J. Phys. Soc. Jpn. 64, 1877 (1995).

${ }^{12}$ H. Kino and H. Fukuyama, J. Phys. Soc. Jpn. 65, 2158 (1996).

${ }^{13}$ H. Seo, J. Phys. Soc. Jpn. 69, 805 (2000).

${ }^{14}$ S. Moroto, K.-I. Hiraki, Y. Takano, Y. Kubo, T. Takahashi, and T. Nakamura, J. Phys. IV 114, 399 (2004).

${ }^{15}$ R. Wojciechowski, K. Yamamoto, K. Yakushi, M. Inokuchi, and A. Kawamoto, Phys. Rev. B 67, 224105 (2003).

${ }^{16}$ T. Kakiuchi, Y. Wakabayashi, H. Sawa, T. Takahashi, and T. Nakamura, J. Phys. Soc. Jpn. 76, 113702 (2007).

${ }^{17}$ Y. Nogami, S. Kagoshima, T. Sugano, and G. Saito, Synth. Met. 16, 367 (1986).

${ }^{18}$ C. P. Heidmann, A. Bansteiner, F. Grob-Alltag, B. S. Chandrasekhar, and E. Hess, Solid State Commun. 84, 711 (1992).

${ }^{19}$ N. A. Fortune, K. Murata, M. Ishibashi, M. Tokumoto, N. Kinoshita, and H. Anzai, Solid State Commun. 79, 265 (1991).

${ }^{20}$ J. Moldenhauer, Ch. Horn, K. I. Pokhodnia, D. Schweitzer, I. Heinen, and H. J. Keller, Synth. Met. 60, 31 (1993).

${ }^{21}$ T. Yamamoto, M. Uruichi, K. Yamamoto, K. Yakushi, A. Kawamoto, and H. Taniguchi, J. Phys. Chem. B 109, 15226 (2005).
${ }^{22}$ N. Tajima, M. Tamura, Y. Nishio, K. Kajita, and Y. Iye, J. Phys. Soc. Jpn. 69, 543 (2000).

${ }^{23}$ V. Železný, J. Petzelt, R. Swietlik, B. P. Corshunov, A. A. Volkov, G. V. Kozlov, D. Schweitzer, and H. J. Keller, J. Phys. (France) 51, 869 (1990).

${ }^{24}$ M. Dressel, G. Gruner, J. P. Pouget, A. Breining, and D. Schweitzer, J. Phys. IV 4, 579 (1994).

${ }^{25}$ M. Watanabe, Y. Noda, Y. Nogami, and H. Mori, J. Phys. Soc. Jpn. 73, 116 (2004).

${ }^{26}$ K. Yakushi, Bull. Chem. Soc. Jpn. 73, 2643 (2000).

${ }^{27}$ Y. Yue, C. Nakano, K. Yamamoto, M. Uruichi, R. Wojciechowski, M. Inokuchi, and K. Yakushi, J. Phys. Soc. Jpn. 78, 044701 (2009).

${ }^{28}$ M. Maksimuk, K. Yakushi, H. Taniguchi, K. Kanoda, and A. Kawamoto, J. Phys. Soc. Jpn. 70, 3728 (2001).

${ }^{29}$ The bending mode of ethylene groups is observable in the spectral region of $1400-1435 \mathrm{~cm}^{-1}$.

${ }^{30}$ Strictly speaking, the linear relationship cannot be applied straightforwardly to the estimation of site charge of charge-rich sites because a dipole-dipole interaction occurs between the phonon modes of charge-rich sites. However, the perturbation of the frequency by this dipole-dipole interaction is very small. This situation is similar to the case of the $\nu_{2}$ mode, in which the frequency is perturbed not only by site charge but also by weak electron-molecular-vibration interaction.

${ }^{31}$ Y. Yue, K. Yamamoto, C. Nakano, M. Uruichi, K. Yakushi, M. Inokuchi, T. Hiejima, and A. Kawamoto, Physica B 405, S232 (2010).

${ }^{32}$ T. Kawai and A. Kawamoto, Phys. Rev. B 78, 165119 (2008).

${ }^{33}$ Y. Tanaka and K. Yonemitsu, J. Phys. Soc. Jpn. 77, 034708 (2008).

${ }^{34}$ The linewidth of the $\nu_{2}^{3}$ mode involves the effect of the hidden $\nu_{2}^{2}$ mode. It is therefore inappropriate to compare the linewidths of the $\nu_{2}^{3}$ of different compounds since the separation between $\nu_{2}^{2}$ and $\nu_{2}^{3}$ differs slightly between $\alpha$-(ET) $)_{2} \mathrm{I}_{3}$ and $\alpha-(\mathrm{ET})_{2} \mathrm{NH}_{4} \mathrm{Hg}(\mathrm{SCN})_{4}$.

${ }^{35}$ R. Kubo, Adv. Chem. Phys. 15, 101 (1969).

${ }^{36}$ W. G. Rothschild, J. Chem. Phys. 65, 455 (1976).

${ }^{37}$ Since the linewidth is much broader than the spectral resolution $\left(2 \mathrm{~cm}^{-1}\right)$, we neglected the effect of the slit function. 
${ }^{38}$ To obtain the second moment of the spectrum, a much wider spectral range should be integrated.

${ }^{39}$ Since the average site charge $\left(\rho_{0}\right)$ which corresponds to $\nu_{2}^{1}$ was $\sim 0.4$, we assumed the distribution function of site charge $(x)$ given by $\frac{1}{\sqrt{2 \pi} \Delta_{c}} \exp \left[-\frac{(x-0.4)^{2}}{\left(\sqrt{2} \Delta_{c}\right)^{2}}\right]$. As the site charge should be in the range of $0<x<1$, the variance was restricted to the range of $3 \Delta_{\mathrm{c}}<\sim 0.4$.

${ }^{40}$ T. Takahashi, Y. Nogami, and K. Yakushi, J. Phys. Soc. Jpn. 75, 051008 (2006).

${ }^{41}$ H. Watanabe and M. Ogata, J. Phys. Soc. Jpn. 75, 063702 (2006).

${ }^{42}$ Similar results were reported by Merino et al. They calculated the dynamical charge susceptibility and charge correlation function using a square lattice model. The collective mode which corresponds to a checkerboard $\mathrm{CO}$ is very softened, when the Coulomb interaction is close to a critical value. J. Merino, A. Greco, R. H. McKenzie, and M. Calandra, Phys. Rev. B 68, 245121 (2003).

${ }^{43}$ The corresponding dip and vibronic $\nu_{3}$ mode was found at 2628 and $1315 \mathrm{~cm}^{-1}$ in $\alpha-\left({ }^{13} \mathrm{C}-\mathrm{ET}\right)_{2} \mathrm{I}_{3}$. Therefore, the isotope shift $\left(60 \mathrm{~cm}^{-1}\right)$ of the dip is nearly twice as large as that $\left(34 \mathrm{~cm}^{-1}\right)$ of the vibronic $\nu_{3}$ mode.

${ }^{44}$ G. Visentini, M. Masino, C. Bellitto, and A. Girlando, Phys. Rev. B 58, 9460 (1998).

${ }^{45}$ M. E. Kozlov, K. I. Pokhondnia, and A. A. Yuruchenio, Spectrochim. Acta, Part A 45, 437 (1989); J. E. Eldridge, C. C. Homes,
J. M. Williams, A. M. Kini, and H. Hau Wang, ibid. 51, 947 (1995).

${ }^{46}$ As to the lattice modes, this compound involves 33 optical phonons which are classified into $3 A_{\mathrm{g}}+12 A_{\mathrm{u}}$ translational modes and $15 A_{\mathrm{g}}+3 A_{\mathrm{u}}$ librational modes, when the space group is $P \overrightarrow{1}$. The $A_{\mathrm{g}}$ mode is Raman active and the $A_{\mathrm{u}}$ mode is infrared active. In the $\mathrm{CO}$ state, however, the selection rule is broken, and all of the lattice modes are detectable in both infrared and Raman spectra.

${ }^{47}$ R. G. Della Valle, A. Brillante, G. Visentini, and A. Girlando, Physica B 265, 195 (1999).

${ }^{48}$ K. I. Pokhodnia, A. Graja, M. Weger, and D. Schweitzer, Z. Phys. B: Condens. Matter 90, 127 (1993).

${ }^{49}$ R. Świetlik, D. Schweitzer, and H. J. Keller, Phys. Rev. B 36, 6881 (1987).

${ }^{50}$ N. Nakaya, K. Itoh, Y. Takahashi, H. Itoh, S. Iwai, S. Saito, K. Yamamoto, and K. Yakushi, Phys. Rev. B 81, 155111 (2010).

${ }^{51}$ M. Meneghetti, R. Bozio, and C. Pecile, J. Phys. 47, 1377 (1986).

${ }^{52}$ The infrared light was focused on the rectangular area of $50 \mu \mathrm{m} \times 200 \mu \mathrm{m}$. The spectrum was taken in the cooling process.

${ }^{53}$ The Raman spectrum was measured with the polarization of $E \| b$ on the (001) crystal face using $\alpha-\left({ }^{13} \mathrm{C}-\mathrm{ET}\right)_{2} \mathrm{I}_{3}$, as the spectrum of metallic phase is very different from that of $\mathrm{CO}$ phase in this condition. 